\title{
TÉCNICAS DE CULTIVO PARA EL CONTROL DE LA EROSIÓN EN LA ZONA SEMIÁRIDA. RESULTADOS PRELIMINARES.
}

\section{SANDRA PERRET D.}

Ingeniero Forestal, U.de Chile. División Silvicultura, Instituto Forestal. Huérfanos 554, Stgo. Chile, fono: 6930700 , fax: 6381286 .

\section{RESUMEN}

Los árboles en los sistemas de producción contribuyen notablemente a proteger el suelo de la erosión, a mantener la fertilidad del suelo, a aumentar la capacidad de infiltración del agua. a proteger los cultivos del sol y el viento y a disminuir los riesgos de plagas y enfermedades. Todo esto, complementado con técnicas de preparación de suelo adecuadas, contribuye a recuperar sistemas degradados.

El presente trabajo pretende determinar la o las altemativas de Sistemas Integrados de Producción que dismintuan los procesos erosivos presentes en ecosistemas semiáridos y que aprovechen al maximo el escaso recurso hidrico. En este articulo se entregan los resultados preliminares en cuanto a la respuesta de la vegetación y a pérdidas de suelo.

Palabras Clave: Cultivos integrados, Erosión

\section{SUMMARY}

Trees have a benefical influence in rural production systems, due to their role in soil erosion control, soil fertility increase, infiltration enhancement in the soil, agriculture crops protection against wind and solar radiation, minimizing pest and diseases risks. Therefore it is espected that degraded areas can be restored by afforestation and proper soil cultivation methods.

Experiences to obtain altemative agroforestry systems in a semiarid environment with lack of rainfall, in order to obtain higher yields in crops with an efficient protection against soil erosion, are analized in this paper. Preliminary results of biomass production and soil erosion impact are given.

Keywords: Agroforestry systems, Erosion. 


\section{INTRODUCCIÓN}

Coquimbo es la Región del país donde es más actual y crítica la desertificación; alrededor de un $20 \%$ de la superficie regional se encuentra calificado en el grado extremo de desertificación. Estas limitaciones se hacen especialmente evidentes en el sector de las Comunidades Agrícolas, las que corresponden a una propiedad común donde el número de habitantes es superior a la potencialidad del medio (IREN, 1978).

La condición de marginalidad de los comuneros agrícolas, es en parte el resultado de un uso y manejo de ecosistemas frágiles sin considerar una planificación que permita la estabilidad socioeconómica en armonia con el medio, lo que se traduce en una relación hombre-naturaleza cada vez más rígida.

La estrategia a seguir para el mejoramiento de estos sistemas contempla acciones sobre cuatro factores fundamentales: El hombre, a través de la capacitación y educación; la vegetación, mediante la recuperación de las formaciones naturales, el uso racional de los recursos existentes y la creación de nuevos recursos; el suelo, por medio de su recuperación, conservación y uso racional, y, finalmente, el agua, recurso escaso y de irregular disponibilidad, cuyo uso debe ser optimizado.

Examinando los fenómenos que tienen lugar en esta región de Chile, el de mayor impacto lo constituye el carácter torrencial de las lluvias, que concentradas en el período invernal potencian la erodabilidad de los suelos.

El balance hídrico en las zonas áridas y semiáridas debe constituir el principal elemento de análisis para el manejo sustentable de los ecosistemas, debido a la escasa disponibilidad de agua y a su gran demanda para diversos usos.

Si se analiza el proceso precipitación - escorrentía, se advierte la gran cantidad de caudal sólido que se transporta, dando origen a fuertes procesos erosivos. Sin embargo, no existe para estas zonas una cuantificación de pérdidas de suelo. Por esto, es fundamental desarrollar investigación que aborde el problema de erosión y pérdida de este recurso, mediante una metodología de cuantificación de pérdidas de suelos y escorrentia bajo diferentes tipos de cultivos.

La cuantificación de las pérdidas de suelo provocadas por la erosión hídrica, bajo diferentes tipos de cultivo, es de importancia para la proposición de acciones de uso de estos ecosistemas.

En el año 1992 se estableció en una pequeña cuenca, cercana a la ciudad de Illapel, un dispositivo experimental para medir las pérdidas de suelo por erosión hídrica, bajo diferentes cultivos y combinaciones de estos, y para evaluar el comportamiento de estos cultivos. 


\section{OBJETIVOS}

El objetivo general de la investigación es la búsqueda de cultivos alternativos, con fines múltiples, que resulten adecuados para las condiciones de suelo y clima de la zona semiárida y permitan mejorar la calidad de vida de sus habitantes.

Los objetivos especificos son en este caso evaluar la respuesta de diferentes cultivos y combinaciones de estos bajo las condiciones climáticas y edáficas locales, en términos productivos, y dimensionar sus efectos de protección ante las pérdidas de suelo por erosión hídrica. A la luz de los resultados obtenidos, sugerir practicas silvoagropecuarias que representen oportunidades de desarrollo para la zona.

En definitiva aportar ideas para disminuir los procesos de desertificación presente en estas zonas y elevar las condiciones de vida de sus pobladores.

\section{MATERIAL Y MÉTODO}

\section{Área de Estudio}

El ámbito de acción de los trabajos corresponde al secano de la IV región, en el área de las Comunidades Agricolas, donde los sistemas productivos son de escasa rentabilidad, debido principalmente a que los terrenos están degradados como producto de la sobreutilización agricola y ganadera.

El área de estudio está centrada en una subcuenca, cuya quebrada principal es afluente de la quebrada "Quelón" en la Comunidad Agrícola de Tunga Norte. Sus coordenadas geográficas son $31^{\circ} 37^{\prime} 35^{\prime \prime}$ de latitud sur y $71^{\circ} 19^{\prime} 30^{\prime \prime}$ de longitud oeste (Carta IGM Mincha, escala 1:50.000).

El terreno elegido corresponde a un área con una sobreexplotación de los recursos, suelos en proceso de degradación, minima cubierta vegetal y fuertes pendientes.

La microcuenca bajo estudio cubre una superficie de 62,5 ha, el ensayo se ubicó en la vertiente noroeste, con exposición predominante sureste. La instalación de los módulos se centró en esta vertiente con el fin de homogeneizar los parámetros de pendiente, exposición y altitud. La pendiente media de estos módulos es de $40 \%$ y la altitud media de $318 \mathrm{msnm}$.

En relación a las propiedades del suelo, estos son de origen volcánico y sedimentario marino, con texturas franco-arcillo-arenosas en superficie, francoarcillosas a medio perfil y arcillosas densas en profundidad. La reacción del suelo ( $\mathrm{pH}$ ) varía de ligeramente ácida a neutra. La relación carbono / nitrógeno es inferior a 15, lo que refleja que son suelos pobres en nitrógeno, y la proporción de calcio y magnesio es mayor en profundidad en el perfil. El área de ocupación de las raices varía entre 80 y $100 \mathrm{~cm}$ de profundidad. 


\section{Base Teórica}

La escorrentía es la parte de la precipitación que se pierde por el flujo superficial o subsuperficial. En general se considera solo la parte de agua correspondiente a la escorrentía superficial, por lo que se cumple que:

$$
E=P-I
$$

Donde:

E : Escorrentia

$P:$ Precipitación

$I$ : Infiltración

La mayoria de los factores que intervienen en el proceso de escorrentía están intimamente relacionados con el proceso de infiltración. Sin embargo, en un suelo ya saturado el volumen y la velocidad del flujo de escorrentia están en función de:

- Volumen e intensidad de las precipitaciones

- Capacidad de almacenamiento superficial del suclo

- Pendiente del terreno

- Rugosidad de la superficie del suelo

De acuerdo a esto la evaluación de la escorrentia está basada en datos climáticos. edáficos y de crecimiento y producción de la vegetación.

La escorrentia total es función de la duración e intensidad de la lluvia. Las lluvias de larga duración, aunque sean de baja intensidad, alteran la estructura superficial del suelo. por lo cual decrece la capacidad de infiltración. Lo mismo ocurre con lluvias de corta duración pero de gran intensidad. pues se provoca con frecuencia un sellado de las capas superficiales, ya que las particulas finas son arrancadas debido al fuerte golpe de las gotas de lluvia (Mintegui y Lopez, 1990).

Con el objetivo de conocer el comportamiento local de las precipitaciones se instaló en el lugar de ensayo una estación meteorológica automática ( Davis Instruments, modelo Monitor II). que previamente se envió a la Dirección Meteorológica de Chile para su verificación, calibración y validación. de acuerdo a las normas internacionales de meteorología.

La estación entrega la siguiente información:

- Temperatura interior y exterior $\left({ }^{\circ} \mathrm{C}\right)$

- Humedad relativa ( \% )

- Presión atmosf́crica ( milibares ) 
- Precipitación ( $\mathrm{mm}$ )

- Dirección del viento

- Velocidad del viento $(\mathrm{km} / \mathrm{h})$

Esta estación es capaz de almacenar 1440 mensajes en intervalos de una hora, por lo cual, según sus especificaciones técnicas. la memoria que trae incorporada podria almacenar sin problemas para este intervalo de frecuencia, aproximadamente 2 meses de información.

La información climática almacenada por la estación se rescata mediante un computador portátil. donde se traspasan los datos mes a mes para su análisis.

\section{Dispositivo Experimental}

Se adecuó una metodología que consiste en el establecimiento de módulos o áreas de comprobación de prácticas conservacionistas, mediante parcelas de escurrimiento. Estas parcelas son de tipo rectangular. con el eje longitudinal orientado en la dirección de la pendiente. Su construcción contempla un marco metálico que encierra cada tratamiento. con el objeto de aislar y concentrar la escorrentía en su extremo más bajo, y un colector para recibir el sedimento acarreado dentro del área de este tratamiento (Figura $\mathrm{N}^{\circ} 1$ ).

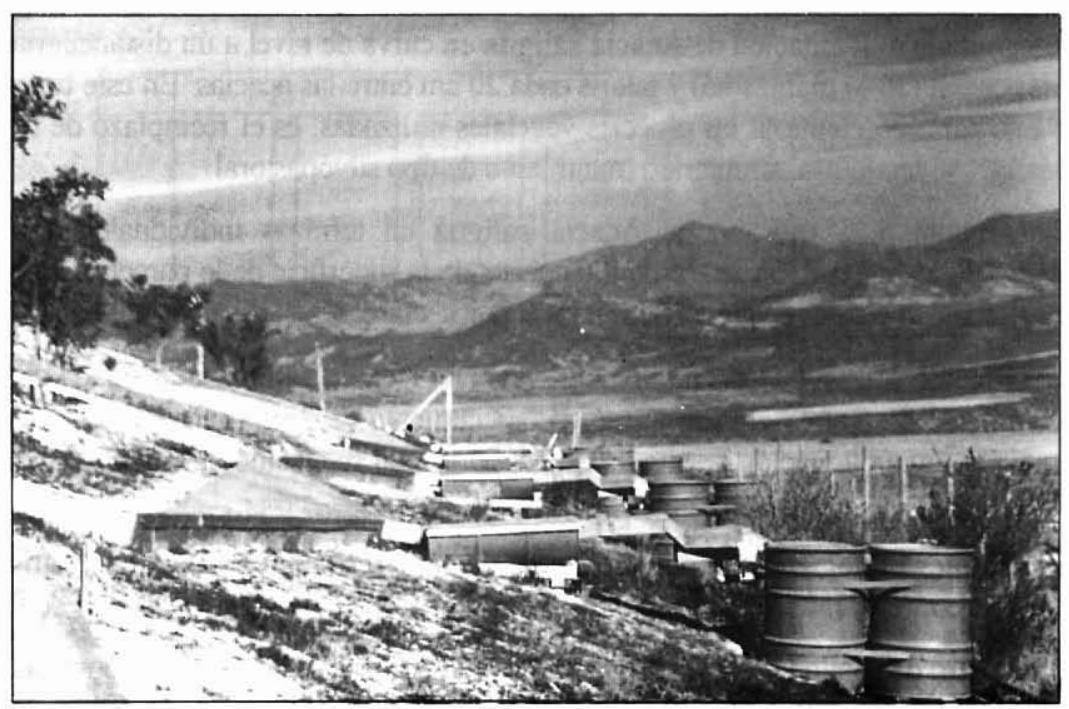

Figura N`1. DISEÑO DE COLECTORFS PARA MEDICIÓN DE EROSIÓN 
La medición de la escorrentía, además de la de los volúmenes de material transportado, se integra a la información climática recogida por la Estación Meteorológica para el análisis del efecto de los tratamientos.

Las parcelas de escorrentia se establecieron en el año 1992 y se incluyeron seis tratamientos que contemplan practicas de cultivo compatibles con las costumbres y tradiciones de los pobladores $\mathrm{El}$ diseño experimental de estos módulos de medición de erosión corresponde a un sistema de bloques. con seis tratamientos y tres repeticiones. El área se protegió debidamente con cercos para evitar el ingreso de animales y personas.

Las variables evaluadas en cada parcela son biológicas y fisicas. en función de los siguientes tratamientos:

Tratamiento 1: Parcela testigo de pradera natural.

Tratamiento 2: Cultivo tradicional (trigo): Este tratamiento corresponde a la siembra de trigo, de acuerdo al uso tradicional en la zona. Para el caso del ensayo se sembró una superficie de $126 \mathrm{~m}^{2}$ con una cantidad de semillas equivalente a $5 \mathrm{~kg}$ (400 kg/ha).

Tratamiento 3: Plantación de Acacia saligna en curva de nivel a un distanciamiento de $4 \mathrm{~m}$ por $1 \mathrm{~m}$ ( 2500 plantas /há.) y falaris cada $20 \mathrm{~cm}$ entre las acacias. Entre curvas (fajas) se sembró trigo y hualputra en una proporción de 81 gr de hualputra por $3 \mathrm{Kg}$ de trigo $(240 \mathrm{Kg} / \mathrm{ha})$. La incorporación de hualputra tiene como finalidad favorecer la captación de nitrógeno e incrementar la productividad del trigo.

Tratamiento 4: Plantación de Acacia saligna en curva de nivel a un distanciamiento de $3 \mathrm{~m}$ por $2 \mathrm{~m}$ ( 1670 plantas/há) y falaris cada $20 \mathrm{~cm}$ entre las acacias. En este tratamiento lo que varia en cuanto a las especies vegetales utilizadas, es el reemplazo de trigo por siembra de hualputra, simulando tratamiento de tipo silvopastoral.

Tratamiento 5: Plantación de Acacia saligna en terrazas individuales de $1 \mathrm{~m}$ de diámetro, en disposición de tresbolillo cubriendo la superficie de la parcela $\left(126 \mathrm{~m}^{2}\right)$ con 23 plantas, lo cual equivale a 1825 plantas por hectárea.

Tratamiento 6: Plantación en curvas de nivel de Acacia saligna intercalada cada $3 \mathrm{~m}$ con hileras de Cassia closiana a un distanciamiento de $3 \mathrm{~m}$ por $1 \mathrm{~m}$. Este tratamiento equivale a una plantación de alta densidad. en la cual se efectuó un tratamiento al suelo de escarificado.

Las variables biológicas contempladas son de carácter cuantitativo y cualitativo. Las cuantitativas contemplan:

- Diámetro de cuello ( $\mathrm{cm}$ ) y Altura ( $\mathrm{m}$ ), para las especies arbóreas y arbustivas.

- Peso verde y Peso seco ( tha ). para las muestras de los pastos.

- Peso ( $\mathrm{kg} / \mathrm{ha}$ ), para la siembra de trigo. 
Las variables cualitativas para las especies arbóreas y arbustivas son las siguientes:

-1 Planta Sana

-2 Rebrote

-3 Tallos Quebrados

4 Replante
-5 Ataque de Hongos

-6 Clorosis

-7 Otros daños

-8 Planta Muerta

Las mediciones para las especies del dosel superior se iniciaron 3 meses después de efectuada la plantación, octubre de 1992, y posteriormente se repiten con una frecuencia de 6 meses, de manera de ver la evolución del crecimiento después de cada fin de temporada.

En relación a las mediciones de hualputra y falaris, las muestras se toman después de la maduración del fruto de manera de conocer la producción de semillas.

Para el caso trigo, la cosecha anual se efectúa en el periodo diciembre - enero.

El diseño y distribución de los tratamientos se muestran en la Figura $\mathbf{N}^{\circ} 2$.

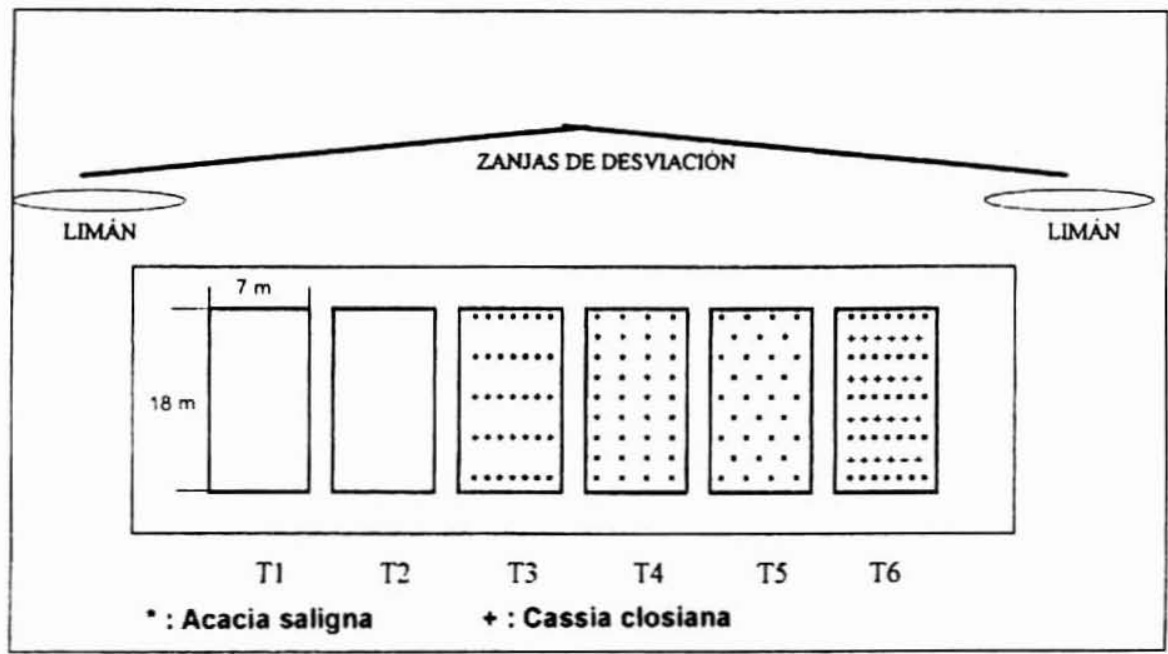

Figura No2. DISEÑO Y DISTRIBUCIÓN DE TRATAMIENTOS.

Las variables físicas contempladas en el diseño son los volúmenes de líquidos y sólidos arrastrados dentro de cada tratamiento.

A la salida de cada marco metálico ( tratamiento ) se ubica en primer lugar una estructura sobre la que se deposita el material de arrastre sólido de mayor tamaño, a continuación sucesivos depósitos capturan el agua de escorrentía. los primeros de los 
cuales almacenan ciertas cantidades de material sólido más fino que se encuentra en suspensión. Tanto los sólidos como el liquido son cuantificados después de cada evento pluviométrico. Para la determinación de los materiales finos en suspensión se envian a laboratorio muestras de un litro para su cuantificación exacta.

Las capacidades en volumen de los depósitos para capturar líquidos y sólidos, fueron inicialmente determinadas mediante una calibración de acuerdo al máximo evento pluviométrico registrado en 24 horas durante los últimos 50 años $(84 \mathrm{~mm})$, según los registros de diferentes estaciones meteorológicas de la zona.

Como es posible apreciar en la Figura $\mathrm{N}^{\circ} 3$, entre los depósitos $\mathrm{C}$ y $\mathrm{E}$ existe un sistema partidor que divide el volumen en 28 partes ( alicuotas ). Este sistema garantiza que cualquier evento pluviométrico por bajo los niveles del máximo antes indicado no excederá la capacidad del dispositivo instalado.

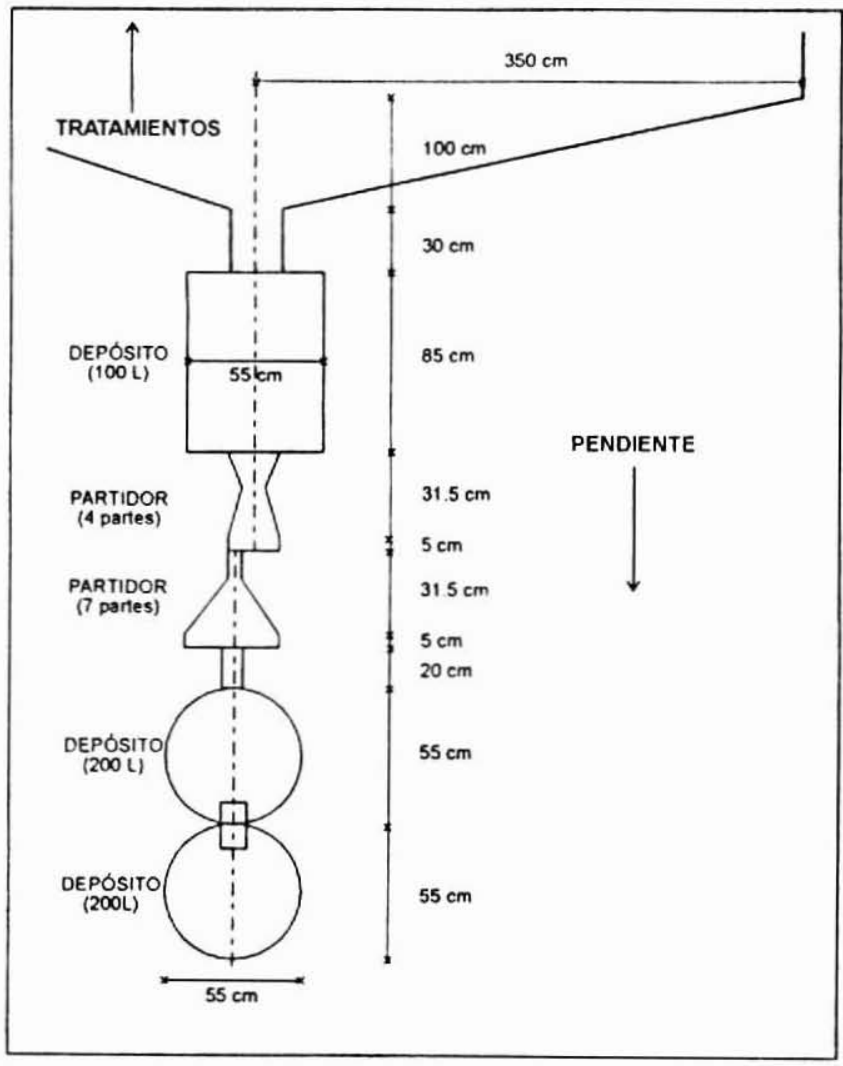

Figura $N^{\circ} 3$. DISEÑO DE COLECTORES PARA MEDICIÓN DE EROSIÓN 


\section{PRESENTACIÓN Y ANÁLISIS DE RESULTADOS}

\section{Información Climática}

La estación meteorológica fue puesta en funcionamiento en el mes de marzo de 1993, a partir del cual se ha rescatado y procesado mes e mes la información climática con un registro horario de todos los parámetros.

En el Cuadro $\mathrm{N}^{\circ} 1$ se muestra un resumen de los valores medios de cada parámetro por mes.

\section{Cuadro $N^{\circ} 1$}

DATOS CLIMÁTICOS 1993

\begin{tabular}{|c|c|c|c|c|c|c|c|c|}
\hline MES & $\begin{array}{l}\text { TEMP. } \\
\text { MIN. } \\
(\cdot C)\end{array}$ & $\begin{array}{l}\text { TEMP. } \\
\text { MAX } \\
\text { (C) }\end{array}$ & $\begin{array}{l}\text { TEMP. } \\
\text { MEDIA } \\
(\cdot C)\end{array}$ & $\begin{array}{c}\text { HUMEDAD } \\
\text { RELATIVA } \\
(\%)\end{array}$ & $\begin{array}{c}\text { PRESIÓN } \\
\text { BAROMÉTRICA } \\
\text { (mb) }\end{array}$ & $\begin{array}{l}\text { VELOCIDAD } \\
\text { VENTO } \\
\text { (km/n) }\end{array}$ & $\begin{array}{l}\text { DIRECCION } \\
\text { VIENTO }\end{array}$ & $\begin{array}{l}\text { PRECIPTTACIONES } \\
\text { (mm) }\end{array}$ \\
\hline MAR & 10,8 & 29,1 & 17.5 & 78,0 & 971,1 & 6,3 & $w$ & 0,0 \\
\hline ABR & 10,4 & 19,9 & 13,7 & 91.2 & 969,4 & 6,6 & $w$ & 68,9 \\
\hline MAY & 4,1 & 25,3 & 12,2 & 79,2 & 973,7 & 3,6 & $w$ & 73,7 \\
\hline JUN & 5,3 & 25,6 & 11,9 & 81,0 & 973.6 & 3,4 & $w$ & 52,0 \\
\hline JUL & 1,4 & 23,4 & 9,6 & 82,9 & 974,3 & 3,6 & $w$ & 0,0 \\
\hline$A G 0$ & 2,7 & 23,8 & 10,9 & 79,7 & 974,0 & 3,8 & $w$ & 38,1 \\
\hline SEP & 5,1 & 23,8 & 11,9 & 80,1 & 973,9 & 5,2 & $w$ & 0,0 \\
\hline OCT & 6,1 & 28,7 & 13,4 & 74,6 & 973,3 & 6,0 & $w$ & 0,0 \\
\hline NOV & 7,5 & 30,6 & 15,4 & 66,5 & 973,5 & 5,6 & $w$ & 0,0 \\
\hline DIC & 9,6 & 25,8 & 16,2 & 70,8 & 971,7 & 5,8 & $w$ & 0,0 \\
\hline
\end{tabular}

El nivel de precipitaciones durante el año 1993, presenta un monto normal, correspondiendo casi exactamente al valor medio registrado por las estaciones meteorológicas de la zona durante los últimos 50 años.

A base de la información reunida por la estación automática instalada en el ensayo, se elaboró un diagrama ombrotérmico ( Figura $\mathrm{N}^{\circ} 4$ ).

Las precipitaciones se concentraron entre los meses de abril y junio, no excediendo los dos dias por mes en cada evento. La última precipitación del año se registró el 29 de agosto con un monto de $38,1 \mathrm{~mm}$, en 12 horas.

La primera lluvia registrada el año 1993 fue de $57,15 \mathrm{~mm}$ y ocurrió el 13 de abril. La máxima intensidad de lluvia registrada en una hora fue de $10,16 \mathrm{~mm}$, el día 7 del mes de mayo. 


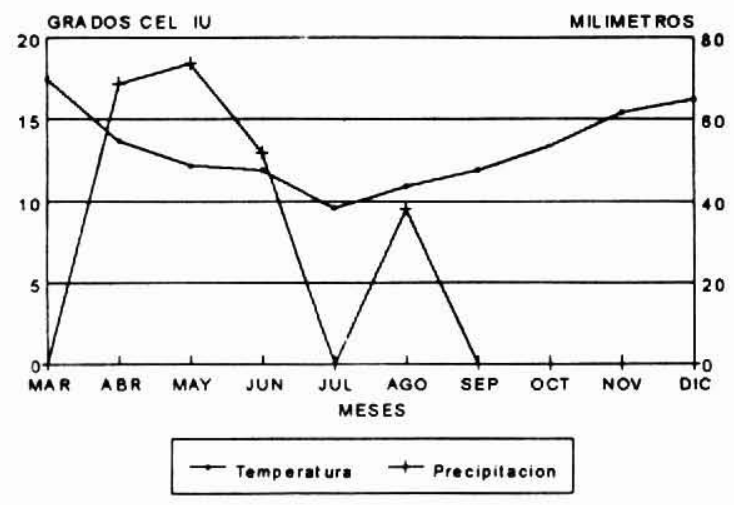

Figura N4. DIAGRAMA OMBROTÉRMICO ESTACIÓN QUELÓN IV REGIÓN.

\section{Vegetación}

Las mediciones para las especies arbóreas. se realizaron 3 meses después de efectuada la plantación, octubre de 1992, las siguientes mediciones se hacen con una frecuencia de 6 meses, de manera de ver la evolución del crecimiento después de cada fin de temporada.

En relación a las mediciones de la hualputra. las muestras se toman después de la maduración del fruto de manera de conocer la producción de semillas y para el caso del trigo, la cosecha se efectúa entre los meses de diciembre y enero de cada año.

Desarrollo de Cultivos y Forraje según Tratamiento.

\section{- Tratamiento 2}

La cosecha de trigo en este caso, fue de $0 \mathrm{~kg}$ para el bloque 1, debido principalmente al daño causado por aves al momento de la siembra y al posterior ataque de roedores al momento de la espigadura. En el caso del bloque 2 y 3 . la cosecha fue de $6,4 \mathrm{~kg}$ y $5 \mathrm{~kg}$, respectivamente. Lo anterior deja de manifiesto. que el terreno no es capaz de producir 
más que lo que se siembra, luego no es un cultivo rentable para estos terrenos con baja fertilidad.

- Tratamiento 3

En el cuadro $\mathrm{N}^{2} 2$ se hace una comparación entre los tratamientos 2 y 3 , en función de la producción de trigo efectuada el año 1992 y cosechada en enero de 1993.

\section{Cuadro $\mathrm{N}^{\circ} 2$}

PRODUCCIÓN DE TRIGO 1993

\begin{tabular}{|l|c|c|c|c|c|c|}
\hline BLOQUE & TRAT. & $\begin{array}{c}\text { SIEMBRA } \\
(\mathbf{k g})\end{array}$ & $\begin{array}{c}\text { SIEMBRA } \\
(\mathrm{kg} / \mathrm{ha})\end{array}$ & $\begin{array}{c}\text { COSECHA } \\
(\mathrm{kg})\end{array}$ & $\begin{array}{c}\text { COSECHA } \\
(\mathrm{kg} / \mathrm{ha})\end{array}$ & $\begin{array}{c}\text { GANANANCIA } \\
(\mathrm{kg} / \mathrm{h})\end{array}$ \\
\hline B1 & T2 & 5 & 396,8 & 0,0 & 0,0 & $-396,8$ \\
& $\mathrm{~T} 3$ & 3 & 238.1 & 6,0 & 476,2 & 238,1 \\
\hline B2 & T2 & 5 & 396,8 & 6,4 & 507,9 & 111,1 \\
& $\mathrm{~T} 3$ & 3 & 238,1 & 8,6 & 682,5 & 444,4 \\
\hline B3 & T2 & 5 & 396.8 & 5,0 & 396,8 & 0,0 \\
& T3 & 3 & 238,1 & 5,0 & 396,8 & 158,7 \\
\hline
\end{tabular}

En el caso del tratamiento 3 (T3) la siembra en la superficie de experimentación $\left(126 \mathrm{~m}^{2}\right.$ ) fue de $3 \mathrm{~kg}$, y en todos los casos se obtuvo una ganancia de producción en comparación al T2 (trigo solo), el cual alcanzó una mínima ganancia de $1,4 \mathrm{~kg}$ en el bloque 2 .

\section{- Tratamiento 4}

La producción promedio de Hualputra. considerando los tres bloques, fue de 5,9 tha, con una proporción de frutos y semillas equivalente al $18 \%$ en relación a la materia seca total. Los resultados obtenidos para cada bloque, en términos de peso seco total y peso de frutos y semilla, se esquematizan en la Figura $N^{\circ} 5$.

Los resultados aquí obtenidos se asemejan bastante a las producciones de esta especie en la zona de Cauquenes VII Región (Ovalle, 1992), donde se obtuvieron en promedio para el primer año de producción 4.6 a $5,4 \mathrm{t} \mathrm{m}$.s./ha/año y, como se observa en la Figura N 5 en el caso de este ensayo en la IV Región la producción de la pradera varió entre 5,4 a $6,5 \mathrm{t} \mathrm{m}$.s./ha/año. Lo que demuestra la importancia de introducir especies leguminosas anuales a los sistema de agricultura de secano. 


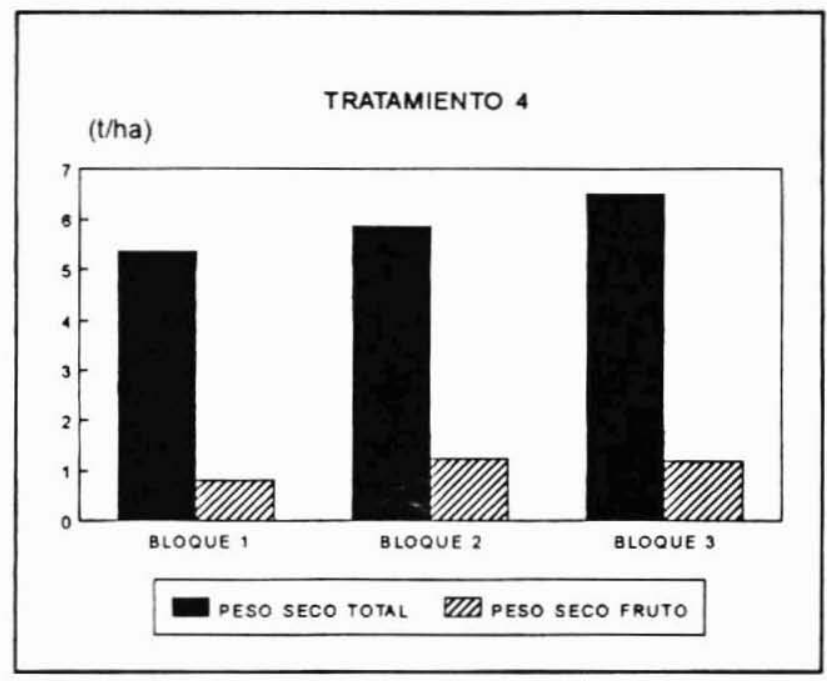

Figura N 5: MATERIA SECA PROMEDIO DE HUALPUTRA

Desarrollo Estrato Arbóreo.

- Sobrevivencia Estrato Arbóreo.

Para el establecimiento de vegetación, tanto arbórea como arbustiva y herbácea, en los tratamientos no se consideró riego de establecimiento, ya que el propósito del ensayo es probar el éxito de las técnicas de preparación de suelo en la respuesta que éstos manifiesten en el prendimiento de las especies seleccionadas, bajo condiciones naturales del sitio.

Tanto en las evaluaciones efectuadas en el primer semestre de establecida la vegetación como en las sucesivas mediciones semestrales, el prendimiento promedio en cada módulo para todos los estratos vegetales considerados no bajo del $93 \%$ (Figura $\mathrm{N}^{\circ} 6$ ). En el Cuadro $\mathrm{N}^{0} 3$ se presenta la supervivencia de la especie Acacia saligna, según tratamiento y bloque a abril de 1994. 


\section{Cuadro No3}

SOBREVIVENCIA DE LA ESPECIE Acacia saligna SEGÚN TRATAMIENTO Y BLOQUE AL 4 DE ABRIL DE 1994.

(\%)

\begin{tabular}{|c|c|c|c|}
\hline TRATAMIENTO & BLOQUE 1 & BLOQUE 2 & BLOQUE 3 \\
\hline T3 & 100.0 & 96,9 & 100,0 \\
\hline T4 & 100,0 & 100,0 & 100,0 \\
\hline T5 & 96,0 & 96,0 & 96,0 \\
\hline T6 & 83.3 & 100,0 & 95,8 \\
\hline
\end{tabular}

La respuesta esperada para la especie Cassia closiana (quebracho) no fue tan satisfactoria. Su supervivencia varia en cada bloque, arrojando un $87,5 \%$ para el bloque 1 , un $100 \%$ en el caso del bloque 2 y un $31,25 \%$ en el bloque 3 .

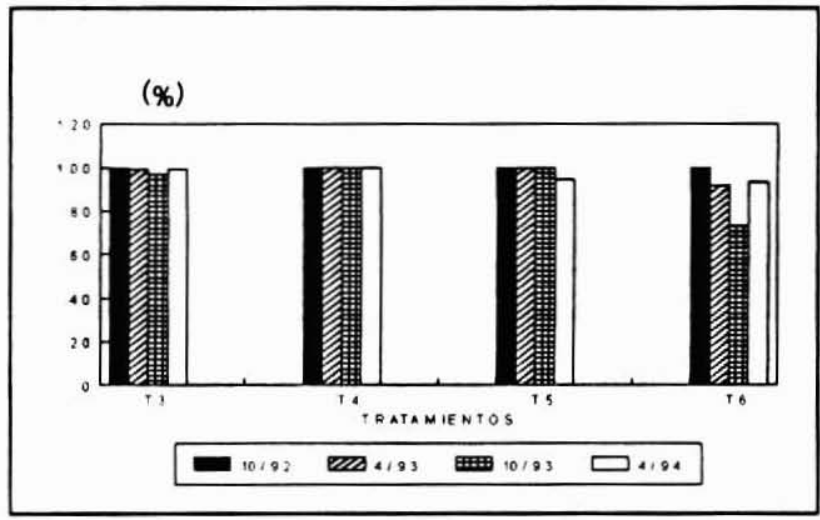

Figura N6: SUPERVIVENCIA Acacia saligna.

Crecimiento.

- Tratamiento 3

En lo que respecta al crecimiento medio observado de la especie Acacia saliga, desde octubre de 1992 a abril de 1994 el incremento en altura ha sido de $0,88 \mathrm{~m}$ y de $1,95 \mathrm{~cm}$ en diámetro de cuello para el bloque 1 . de $1,00 \mathrm{~m}$ y $2,13 \mathrm{~cm}$, respectivamente para el bloque $2, y$ de $1 \mathrm{~m}$ y de $2.28 \mathrm{~cm}$. respectivamente para el bloque 3 . Lo anterior 
demuestra el rápido crecimiento de esta especie aún en condiciones adversas. (Figura No7).

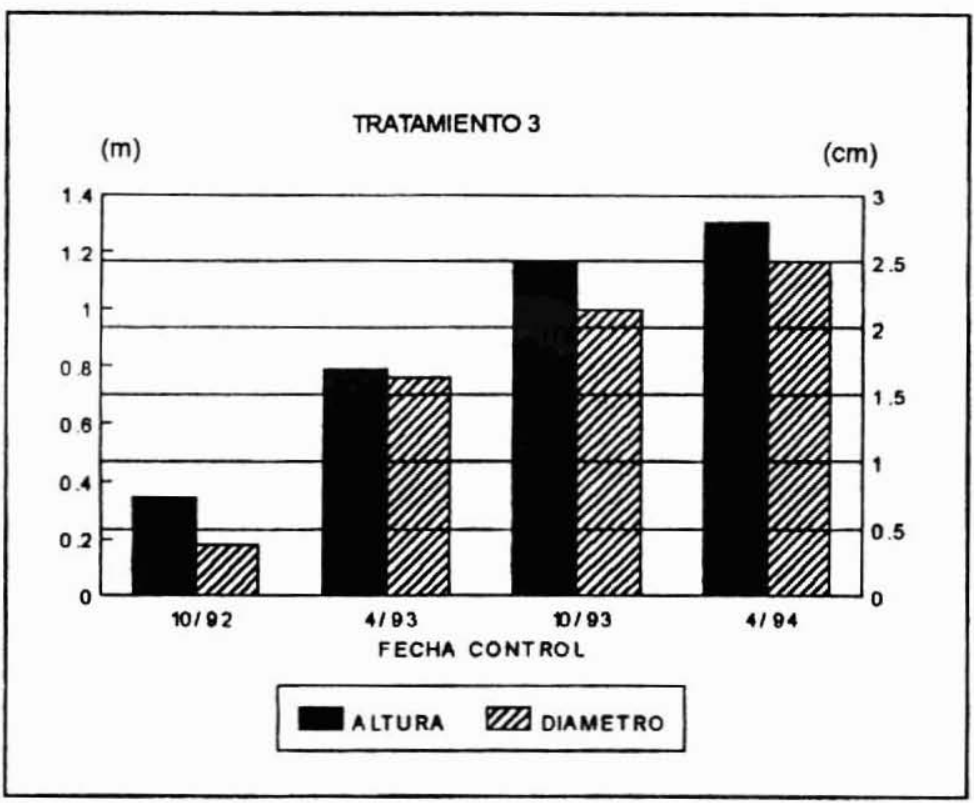

Figura N07: CRECIMIENTO EN ALTURA Y DIAMETRO Acacia saligna

\section{- Tratamiento 4}

El crecimiento observado en el tratamiento 4 para la especie Acacia saliga, según bloque en altura y diámetro de cuello. se indica en el Cuadro $\mathrm{N}^{\circ} 4$.

\section{Cuadro $\mathrm{N}^{\circ} 4$}

CRECIMIENTO ANUAL DE. Acacia saliga EN DAC Y ALTURA, SEGÚN TRATAMIENTO 4.

\begin{tabular}{|l|c|c|c|}
\hline PARAMETRO & BLOQUE 1 & BLOQUE 2 & BLOQUE 3 \\
\hline DAC (cm/año) & 2.17 & 2.17 & 2,35 \\
\hline ALTURA (m/año) & 0.90 & 1.00 & 0.98 \\
\hline
\end{tabular}




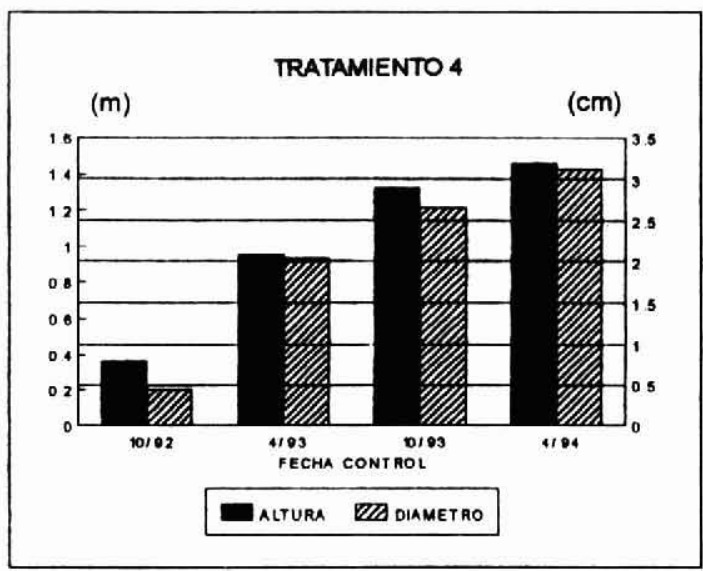

Figura N'8: CRECIMIENTO EN ALTURA Y DIÁMETRO Acacia saligna

- Tratamiento 5

En cuanto al crecimiento de la especie en este tratamiento, los resultados indican que el incremento en altura y diámetro de copa para el bloque 1 fue de $0,91 \mathrm{~m} / \mathrm{año}$ y de $1,91 \mathrm{~cm} /$ año, respectivamente: para el bloque 2 el incremento en altura fue de 0,95 $\mathrm{m} /$ año y de $1,91 \mathrm{~cm} /$ año, y para el bloque 3 de $1,04 \mathrm{~m} /$ año en altura y $2,18 \mathrm{~cm} /$ año en diámetro de cuello (Figura $\mathrm{N}^{\circ} 9$ ).

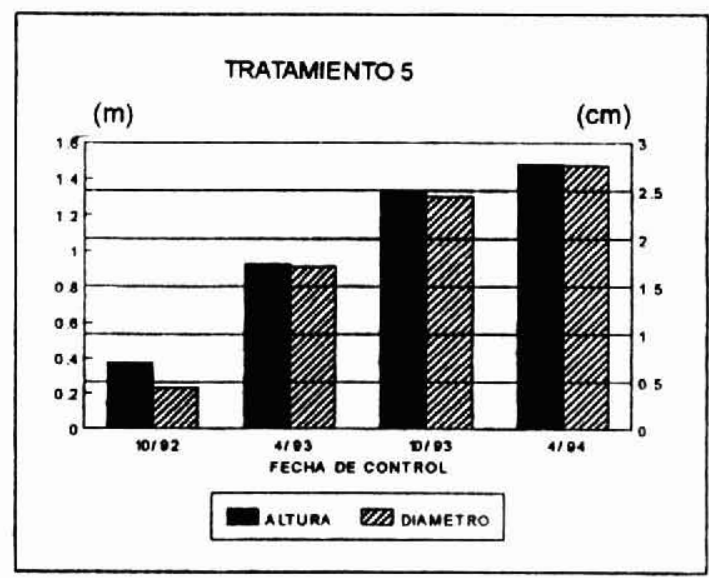

Figura N ${ }^{\circ}$ : CRECIMIENTO EN ALTURA Y DIAMMTRO Acacia saligna 


\section{- Tratamiento 6}

Los incrementos medios de la especie, en relación a la altura y diámetro de cuello para este tratamiento fueron de $0,89 \mathrm{~m} /$ año y de $1,92 \mathrm{~cm} /$ año para el bloque 1 , de 0,78 m/año y $1,72 \mathrm{~cm} /$ año, respectivamente para el bloque 2 y de un 0,82 m/año y 1,67 m/año para el bloque 3. Los resultados semestrales medidos se muestran en la Figura $\mathrm{N}^{\circ} 10$.

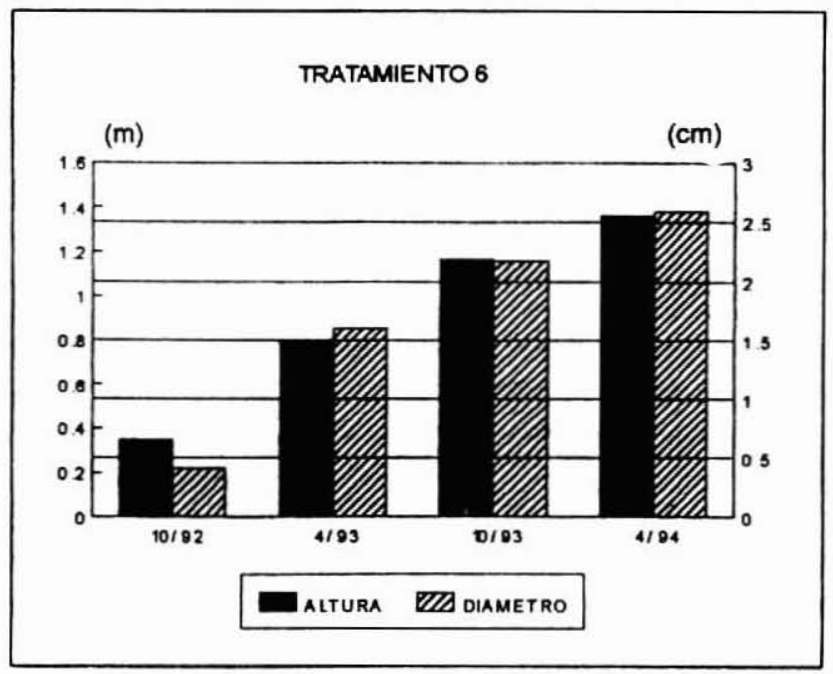

Figura $\mathrm{N}^{\circ} 10$. CRECIMIENTO EN ALTURA Y DIAMETRO, Acacia saligna

En relación al crecimiento de la especie Cassia closiana, incorporada en el tratamiento 6 , el incremento medio alcanzado fue de $0,39 \mathrm{~m} / \mathrm{año}$ en altura y de 0,92 $\mathrm{cm} /$ año en diámetro de cuello para el bloque 1 ; de un $0,24 \mathrm{~m} / \mathrm{año}$ y $0,72 \mathrm{~cm} /$ año para el bloque 2 , y de un $0.13 \mathrm{~m} /$ año y $0.4 \mathrm{~cm} /$ año. respectivamente para el bloque 3 . Los resultados de las mediciones efectuadas se entregan en la Figura $\mathrm{N}^{\circ} 11$.

Comparación entre Tratamientos.

Al efectuar un análisis comparativo entre los 4 tratamientos que contemplan a la especie Acacia saligna, los mejores resultados a la fecha son los del T4 y T5, es decir el que contempla un mayor cubrimiento de la superficie y el de terrazas individuales, respectivamente. 


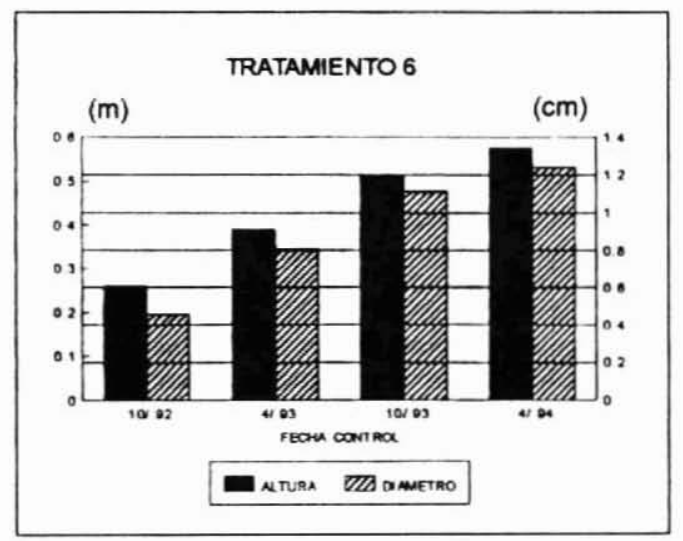

\section{Figura No11. CRECIMIENTO EN ALTURA Y DIȦMETRO Cassia closiana}

El comportamiento en estos tratamientos en los tres bloques fue de un $100 \%$ de supervivencia. La altura promedio a octubre de 1993 es de $1,29 \mathrm{~m}$ para el T4 y de 1,28 $\mathrm{m}$ en T5, y de $2,58 \mathrm{~cm}$ de diámetro de cuello para el T4 y de $2,35 \mathrm{~cm}$ para $\mathrm{T} 5$ en el bloque 1. Para el bloque 2 la respuesta fue relativamente superior con una altura promedio de $1,35 \mathrm{~m}$ en ambos tratamientos y un crecimiento diametral de $2,62 \mathrm{~cm}$ en $\mathrm{T} 4$ y $2,37 \mathrm{~cm}$ en T5. El resultado para el bloque 3 fue de $1,33 \mathrm{~m}$ en T4 y $1,39 \mathrm{~m}$ en T5 de crecimiento en altura y de $2,8 \mathrm{~cm}$ en T4 y $2.61 \mathrm{~cm}$ en T5 en crecimiento diametral. Los resultados para los tratamientos se grafican en la Figura $\mathrm{N}^{\mathrm{N}} 12$.

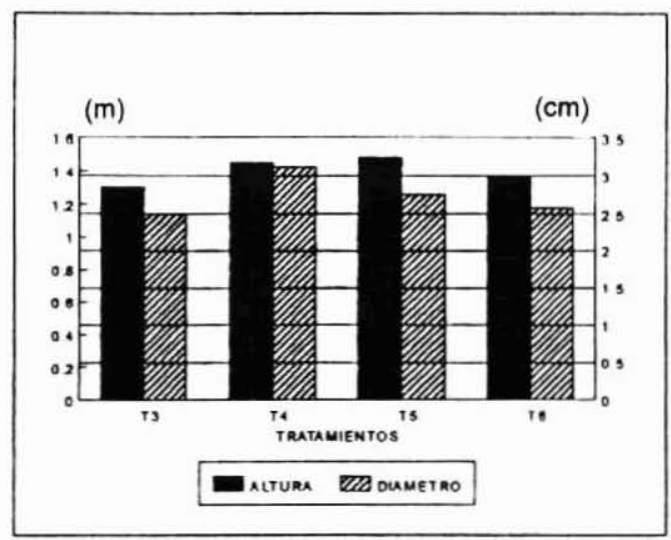

Figura $\mathrm{N}^{\circ}$ 12: CRECIMIENTO EN ALTURA Y DIAMETRO Acacia saligna 


\section{Pérdida de Suelo y Escorrentía.}

Se registró la presencia de escorrentía en los depósitos de almacenamiento de cada tratamiento después de dos eventos pluviométricos.

En la Figura $\mathrm{N}^{\circ} 13$ se aprecia la cantidad total acumulada por bloque y tratamiento, de sólidos y agua registrada en cada medición, según evento pluviométrico. De los valores de pérdidas se observa, que la mayor acumulación se produjo con los eventos pluviométricos del mes de mayo, cuya escorrentía ocupo la capacidad de los dos primeros depósitos de acumulación, lo cual no ocurre en los posteriores eventos (junio y agosto).

A continuación se gráfica el comportamiento de la escorrentia después de cada evento muestreado (Figura $\mathrm{N}^{\circ} 13$ ).

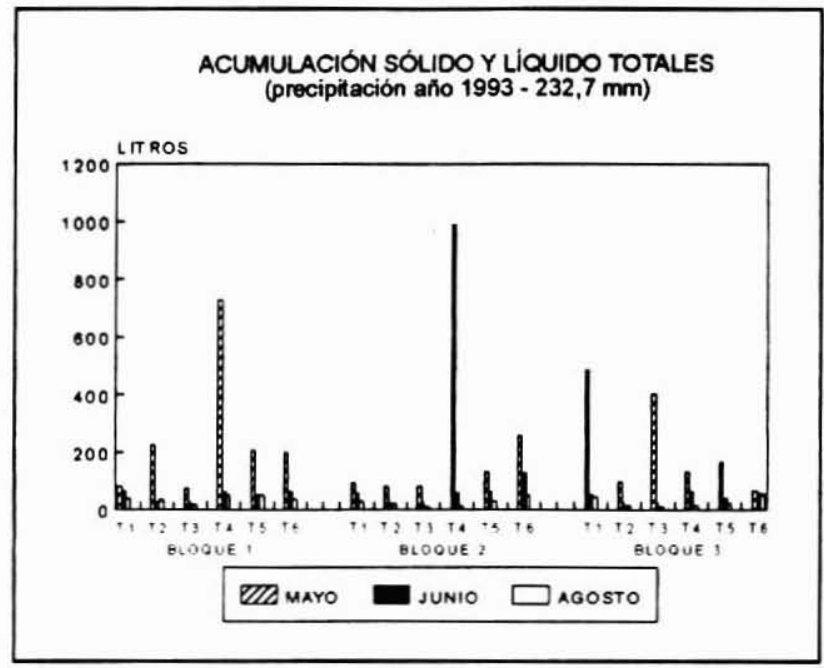

Figura $\mathrm{N}^{\circ} 13$. MUESTRA DE SÓlidOS Y LíQuidos TOTALES

Entre los meses de abril y mayo se registró una precipitación de $142.6 \mathrm{~mm}$ y los dos primeros eventos pluviométricos ocurridos en el mes de abril no fueron suficientes como para acumular una cantidad de agua para tomar muestras. de tal manera que se espero a que se registrara otro evento pluviométrico para efectuar las mediciones correspondientes. Es así como la cantidad de lluvia recibida en estos dos meses arrojó pérdidas de agua y sólido que para los casos del bloque 1 y 2 fue mayor en el tratamiento 4 , sin embargo en el bloque 3 la mayor pérdida se produjo en el tratamiento 1 (Testigo) y el Tratamiento 3. 
La explicación para estas pérdidas es que en ambos tratamientos (T3 y T4) las hileras entre los surcos se encontraban sin vegetación, en el primer caso porque el terreno estaba siendo preparado para la siembra de trigo y, en el segundo, porque aún no emergía la semilla de hualputra. En el caso del testigo en el bloque 3 no existe una explicación lógica, ya que durante el primer año este tratamiento ha estado excluido y se ha recuperado la pradera natural.

En los siguientes eventos pluviométricos las pérdidas disminuyeron considerablemente, producto de que en todos los tratamientos la superficie estaba cubierta en casi un $70 \%$. Las lluvias registradas en los meses de junio y agosto fueron de $52 \mathrm{~mm}$ y $38,1 \mathrm{~mm}$, respectivamente.

En el Cuadro $\mathrm{N}^{\circ} 5$ se presenta el total de agua escurrida para el año 1993, según tratamiento y bloque.

\section{Cuadro No5}

VOLUMEN DE AGUA TOTAL 1993

(L)

\begin{tabular}{|c|c|r|r|}
\hline TRATAMIENTOS & BLOQUE 1 & BLOQUE 2 & BLOQUE 3 \\
\hline T1 & 185.43 & 179.01 & 586,82 \\
\hline T2 & 276.10 & 120.52 & 120.00 \\
\hline T3 & 105.05 & 95.83 & 412.89 \\
\hline T4 & 838.15 & 1054,19 & 202,27 \\
\hline T5 & 304,61 & 224.26 & 228,68 \\
\hline T6 & 296,62 & 435.20 & 178.59 \\
\hline
\end{tabular}

Este último cuadro corresponde a la escorrentía que se produjo, después de cada evento muestreado. Luego el análisis siguiente fue determinar la infiltración de agua en el perfil del suelo. En base a la información obtenida por la estación metereológica, más la obtenida por los dispositivos de control. se obtuvo el porcentaje final de escorrentía según cada tratamiento (Cuadro $\mathrm{N}^{\circ} 6$ ) y por diferencia el porcentaje total de infiltración (Cuadro $\mathrm{N}^{\circ} 7$ ).

\section{Cuadro $\mathrm{N}^{\mathrm{O}} 6$}

ESCORRENTIA TOTAL 1993

(\%)

\begin{tabular}{|c|c|c|c|}
\hline TRATAMIENTOS & BLOQUE 1 & BLOQUE 2 & BLOQUE 3 \\
\hline T1 & 0.76 & 0.67 & 1,50 \\
\hline T2 & 0.74 & 0.39 & 0.32 \\
\hline T3 & 0.33 & 0.24 & 0,83 \\
\hline T4 & 2.02 & 2.20 & 0.63 \\
\hline T5 & 0.98 & 0.78 & 0.67 \\
\hline T6 & 0.94 & 1.47 & 0,80 \\
\hline
\end{tabular}




\section{Cuadro $\mathrm{N}^{\circ} \mathrm{7}$ \\ INFILTRACIÓN TOTAL 1993}

(\%)

\begin{tabular}{|c|c|c|c|}
\hline TRATAMIENTOS & BLOQUE 1 & BLOQUE 2 & BLOQUE 3 \\
\hline T1 & 99,24 & 99,33 & 98,50 \\
\hline T2 & 99,26 & 99,61 & 99,68 \\
\hline T3 & 99,67 & 99,76 & 99,17 \\
\hline T4 & 97,98 & 97,80 & 99,37 \\
\hline T5 & 99,02 & 99,22 & 99,33 \\
\hline T6 & 99,06 & 98,53 & 99,20 \\
\hline
\end{tabular}

Los resultados finales de pérdida de suelo según tratamiento se presentan en el Cuadro No8 extrapolados a la hectárea, para las tres mediciones efectuadas (mayo, junio $y$ agosto), dando en promedio pérdidas que no superan los 0,4 tha/año, lo cual refleja que los tratamientos propuestos como alternativas económicas para la zona son viables hasta la fecha (Cuadro $\mathrm{N}^{\circ}$ ). Lo que se esperaría en los siguientes años es que las pérdidas cada vez fueran de menor magnitud a pesar de que los eventos pluviométricos fuesen más torrenciales.

\section{Cuadro No8}

PERDIDAS DE SUELO AÑO 1993

(kg/ha/aho)

\begin{tabular}{|c|c|c|c|c|}
\hline TRATAMIENTOS & BLOQUE 1 & BLOQUE 2 & BLOQUE 3 & PROMEDIO \\
\hline T1 & 47,20 & 24,10 & 60,50 & 43,93 \\
\hline T2 & 153,00 & 61,50 & 39,20 & 84,56 \\
\hline T3 & 70,50 & 21,20 & 89,90 & 60,53 \\
\hline T4 & 373,20 & 395.10 & 92,60 & 286,97 \\
\hline T5 & 48,20 & 43,40 & 16,30 & 35,97 \\
\hline T6 & 48,80 & 50.90 & 51.70 & 50,47 \\
\hline
\end{tabular}

\section{CONCLUSIONES}

-Durante el año 1993 se presentaron 6 lluvias, distribuidas entre los meses de abril a agosto, con un monto total de $232 \mathrm{~mm}$. La intensidad horaria en éstos eventos no superó los $10 \mathrm{~mm}$. dicho valor se considera poco significativo como para provocar riesgos de erosión hidrica.

-El comportamiento de la escorrentía superficial es decreciente comparando las lluvias de abril a agosto. lo que tiene su explicación en el crecimiento y desarrollo de la vegetación.

-La escorrentía el año 1993 alcanza su mínimo valor en el tratamiento T3, en cada una de las mediciones efectuadas, y el valor máximo se presenta en el tratamiento T4. 
-La infiltración total obtenida en este periodo es en todos los tratamientos cercana al $100 \%$, siendo el valor mínimo registrado de $97,8 \%$.

-En términos de pérdidas de suelo el mayor valor registrado es de 0,37 ton/ha/año y el mínimo de 0,047 tha/año. Estos valores son considerados poco significativos, lo que indica que los tratamientos son efectivos en términos de conservación de suelo, incluso el testigo, el cual por estar excluido ha recuperado su cubierta vegetal.

-Se constata en este año y medio de evaluación el tratamiento T3 (Asociación trigo/Hualputra con acacia y falaris entre surcos) es el que ha demostrado ser el más eficiente en cuanto al aprovechamiento de agua. El tratamiento T4 (Asociación hualputra con acacia y falaris entre surcos), sin embargo, es el que arroja las mayores pérdidas en términos de agua y sólido. Esto tiene su explicación, dado que al momento de producirse las primeras lluvias aún no habian emergido las primeras plantas de hualputra, por lo cual el suelo se encontraba expuesto a la acción erosiva del agua.

-En relación al crecimiento de las especies arbóreas en los diferentes tratamientos, en general ha sido satisfactoria, a excepción de la especie nativa Cassia closiana, la cual alcanzó en uno de los bloques, a mayo de 1994, un $12 \%$ de supervivencia. De lo anterior se puede inferir, que la adaptividad de una especie nativa en terrenos degradados seria más dificil, producto de la mayor exigencia en los requerimientos de nutrientes y agua para su supervivencia.

-En relación a la Acacia saligna, ésta ha demostrado ser una especie de gran adaptabilidad, incluso sometida al estres hídrico del periodo estival. La mejor respuesta en crecimiento y desarrollo de esta especie ha sido en los tratamientos T4 y T5.

-En el establecimiento y producción de las especies herbáceas se aprecia un éxito en el prendimiento de la hualputra y un incremento en la producción en relación al primer año. Los valores obtenidos el año 1993 varian entre 5,4 y 6,5 tha/año, valores que son superiores a los obtenidos el primer año de producción en la estación del INIA en Cauquenes.

-Con respecto al falaris, el incremento en materia seca promedio en relación a su primer año de producción ha sido de 1.7 a 2,2 tha/año, para el tratamiento T4, y de 3,4 a 7,4 tha/año, para el tratamiento T3.

-Para finalizar se debe indicar en relación a la producción de trigo en los tratamientos T2 y T3. En el T3 se cosecho más del doble de lo que se sembró. En trigo solo (T2), solo en el mejor de los casos se recuperó lo sembrado.

\section{REFERENCIAS BIBLIOGRÁFICAS}

Del Pozo, A; Ovalle, C. y Avendaña, J., 1989. Los Medicagos Anuales III. Perspectivas Agronómicas en el Secano Interior de la Zona Mediterránea de Chile. Agricultura Técnica (En prensa). 
INFOR, 1976. Tablas y Factores de Conversión. Manual N7.Santiago, Chile. 97 páginas.

IREN., 1977. Estudio de las Comunidades Agricolas IV Región. Uso y Manejo Actual. Instituto de Investigación de Recursos Naturales. Corporación de Fornento de la Producción, Publicación 20. Santiago, Chile, 54 páginas.

IREN-CORFO., 1978. Estudio de las Comunidades Agricolas de la IV Región. Santiago, Chile volúmenes 9 y 10 (publicación 20).

Mintegui,A.J; Lopez,U.F., 1990. La Ordenación Agrohidrológica en la Planificación. Departamento de Agricultura y Pesca, Gobiemo Vasco. Primera parte, 172 páginas.

Ovalle, C., 1992. Caracterización y Selección de Medicago polymorpha (Hualputra) y otras Leguminosas Forrajeras Anuales, para la Zona Mediterránea de Chile. Programa de Praderas de Secano. INIA. Santiago. 8 páginas. 